\title{
Abhandlungen
}

\author{
Marc Baumgartner, Ivars Udris
}

\section{Call Center ist nicht gleich Call Center}

Personalselektion und -entwicklung in einer dynamischen Branche (auch in der Schweiz)

\begin{abstract}
Untersuchungen in 14 Schweizer Call Centers erbrachten vier Call Center-Typen, die sich hinsichtlich Arbeitstätigkeiten und Kommunikationsrichtung voneinander unterscheiden: (a) Beratungs- und Beschwerdemanagement, (b) Informationsmanagement, (c) Auftragsmanagement und (d) Kunden- und Kampagnenmanagement. Dies hat auch Auswirkungen auf die Personalstruktur, -selektion und -entwicklung der Call Center. Es wird der Frage nachgegangen, welche Kompetenzanforderungen in den unterschiedlichen Call Center-Typen an die Call Center AgentInnen gestellt werden, und wie sich die Rekrutierungspraktiken sowie die Personalentwicklungsmaßnahmen wie Ausbildung, Weiterbildung und Coaching unterscheiden. Ebenfalls aufgezeigt werden Perspektiven für die Zukunft der Call CenterArbeit.
\end{abstract}

\section{$1 \quad$ Einleitung}

Ende der neunziger Jahre erlebte der Call Center-Markt in Europa einen regelrechten Boom. Ziel der Branche war die organisatorische Zusammenlegung von Telefonarbeitsplätzen in Verbindung mit informations- und kommunikationstechnischer Unterstützung zur Verbesserung der Kundenkontakte bei gleichzeitiger Optimierung der Wirtschaftlichkeit. In der Schweiz lagen die Wachstumsraten zwischen 30-40\% in Deutschland sogar bei 50\%. Nach den Jahren des Wachstums hat sich jedoch die Euphorie der Gründerzeit zu Beginn des neuen Jahrhunderts bereits etwas gelegt. Bis ins Jahr 2007 dürfte die Branche in der Schweiz jährlich noch um 6\% zulegen. Damit liegt sie unter dem europäischen Durchschnitt von 7,1\% (www.persoenlich.com). Trotzdem nehmen die Gesprächsvolumen weiterhin zu und dadurch auch die Belastung der Call Center Mitarbeitenden sowie der Unmut der Kunden über die oft erheblichen Qualitäts- und Leistungsmängel bei der telefonischen Kundenbetreuung. Call Center und deren Arbeitsbedingungen sind somit nach wie vor von öffentlichem Interesse und daher Gegenstand arbeitswissenschaftlicher Untersuchungen. Die in Call Center aus der Arbeitssituation resultierenden Beanspruchungen sowie die wichtigsten Belastungsfaktoren waren in Deutschland und der Schweiz im Rahmen zahlreicher Projekte Gegenstand der

Arbeit, Heft 1, Jg 14 (2005), S.3-17 
Untersuchungen(Dormann/Zapf/Isic 2003; Metz/Rothe/Degener 2001; Scherrer 2001; Richter/Debitz/Schulze 2002; Timm 2003; Grebner/ u.a. 2003).

Oft wird die strategische Bedeutung eines Call Centers für den Unternehmenserfolg zwar gesehen, jedoch nach wie vor unterschätzt. Denn es stellt sich die Frage, warum gerade dieser bedeutende Bereich der Kundenbetreuung entweder an externe Dienstleister abgetreten wird oder firmeninterne Abteilungen gebildet werden, deren Hauptaufgabe darin besteht, sich mit den „lästigen“ Kundenfragen auseinander zu setzen, damit sich die Mitarbeitenden der anderen Abteilungen ungestört den „wichtigen“ Aufgaben des Unternehmens zuwenden können. Die geringe Anerkennung der Call Center und deren Mitarbeitenden in einer Organisation haben einen entsprechenden Einfluss auf die hohen Fluktuationsraten und Fehlzeiten. Als weitere Fluktuationsgründe werden mangelnde Entwicklungsmöglichkeiten, Monotonie sowie persönliche Veränderungen wie z. B. das Interesse, etwas anderes kennen zu lernen, Schwangerschaft oder Beendigung einer Ausbildung (Baumgartner/Good/Udris 2002) genannt. Die Arbeit in einem Call Center (CC) wird somit oft als Übergangslösung gesehen. Wenig herausfordernde Aufgaben, die nur geringes Potential zur persönlichen Weiterentwicklung bergen, gekoppelt mit geringen Handlungs- und Kontrollspielräumen sowie schlechten, überlasteten Führungsstrukturen, führen in vielen Fällen zur Kündigung (Timm 2003). Die durch Fluktuationen entstehenden Rekrutierungs- und Qualifizierungskosten, der Wissensverlust und der zusätzlich benötigte Führungsaufwand rechtfertigen sicherlich höhere Investitionen in Mitarbeiterbindungs- und Kompetenzentwicklungsmaßnahmen. Dieser Thematik nahm sich die Studie „CALL: Call Centers in der Schweiz“ an, deren Teilergebnisse in der Folge präsentiert werden sollen (Baumgartner u.a. 2002; Baumgartner/Udris 2003, 2004a, 2004b; Udris/Baumgartner 2003). In dieser Studie wurden 14 CC in der deutschsprachigen Schweiz, die ein breites Spektrum der vielfältigen Branche abdecken, untersucht. Dabei fanden Interviews mit 13 Call Center ManagerInnen(CCM) und 20 Call Center TeamleiterInnen(CCT) statt. Zudem haben 243 Call Center Agenten (CCA) einen schriftlichen Fragebogen ausgefüllt.

\section{Call Center Typen}

Call Centers werden meist anhand der Kommunikationsrichtungen Inbound-Call Center vs. Outbound-Call Center oder nach deren organisatorischen Zugehörigkeit Inhouse-Call Center vs. externe Call Center-Dienstleister differenziert. Zwischen den verschiedenen Call Center bestehen jedoch Unterschiede in Bezug auf deren Auftrag, Mitarbeiterstruktur, Branchenzugehörigkeit, technischen Entwicklungsstand, Kundensegment etc. Einzig die Tatsache, dass sie eine telefonische Dienstleistung gegenüber Kunden erbringen, ist allen gemeinsam.

Da die Aufgaben und Tätigkeiten, welche ausgeführt werden, sich zum Teil stark voneinander unterscheiden, ermöglicht eine typologische Betrachtungsweise, jenseits der üblichen Unterteilung in Inbound- vs. Outbound-Call Center, wesentlich differenziertere Ergebnisse, die auf das Personalmanagement, die Qualität der Arbeitstätigkeit und die soziale Situation in den Call Centern verweisen. Daher liegen gegenwärtig mehrere Versuche vor, die Branche in sinnvolle Typen zu differenzieren, welche unterschiedliche Aspekte der Call Center-Arbeit zu berücksichtigen versuchen (Geier/Groh/Grass 2001; Brasse u.a. 2002; Timm 2003). Im Projekt CALL war es den Autoren ebenfalls ein Anliegen, der Unterschiedlichkeit der Branche Rechnung zu tragen (Udris/Baumgartner 2003; Baumgartner/Udris 2004b).

Die vier Call Center-Typen, als Resultat statistischer Kategorisierung der CCA-Tätigkei- 
ten mittels Clusteranalysen, sind: Beratungs- und Beschwerdemanagement (BBM), Informationsmanagement (IM), Auftragsmanagement (AM), Kunden- und Kampagnenmanagement (KKM).

Dabei ist zu berücksichtigen, dass in jedem Typ jeweils auch Tätigkeiten aus den anderen Call Center-Typen ausgeführt werden - jedoch zu einem geringeren Prozentanteil am gesamten Telefonarbeitsvolumen. (In der Folge werden meist die Abkürzungen der Typen verwendet).

\subsection{Beratungs- und Beschwerdemanagement}

Der Typ BBM widmet sich der intensiven Telefonberatung, dem Führen von Hotlines, dem Kundenservice für erworbene Produkte und Dienstleistungen sowie dem Management von Reklamationen und Beschwerden. Die Nebentätigkeiten sind der Verkauf von Produkten und Dienstleistungen bzw. die Auftragsannahme und deren Verarbeitung sowie die Verarbeitung standardisierter Informationen. Es werden vorwiegend Inbound Anrufe erledigt (95\%). Von allen vier Typen dauern die Telefongespräche in diesem Typ signifikant am längsten (durchschnittlich 8,1 Minuten). So können punktuelle oder längerfristige Beziehungen mit den KundInnen entstehen, obgleich das System grundsätzlich auf der nicht an Personen gebundenen Wissensvermittlung und Begleitung basiert. Die Ganzheitlichkeit der Aufgabe und der Tätigkeitsspielraum werden als mittelmäßig aber signifikant größer wahrgenommen als im Typ Informationsmanagement. Drei der 14 Call Center gehören zum Typ BBM.

\section{Abb. 1: Prozentanteile der Tätigkeiten für den Typ BBM}

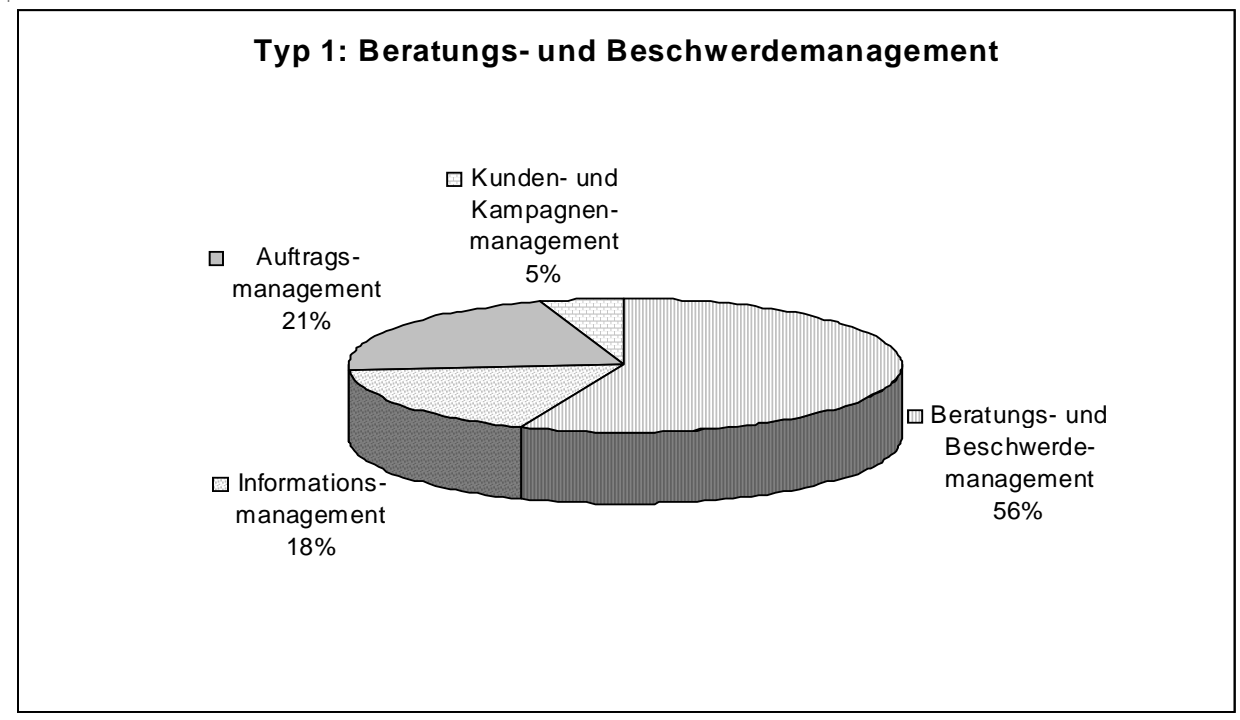

\subsection{Informationsmanagement}

Im Typ IM geht es in erster Linie um die Vermittlung oder Verarbeitung standardisierter Informationen, wie Führen von Adressverwaltungen oder die Entgegennahme und Weiterlei- 
tung von Anrufen. Die Nebentätigkeiten sind das BBM (21\%) sowie das AM (8\%) und das KKM (8\%). Es werden vorwiegend Inbound-Anrufe erledigt (92\%). Von allen vier Call Center-Typen dauern die Telefongespräche in diesem Typ am kürzesten (durchschnittlich 2,5 Minuten). Hier handelt es sich um einen kurzen und einfachen Service, den das Call Center anbietet. Die Ganzheitlichkeit der Aufgabe und der Tätigkeitsspielraum werden dementsprechend signifikant niedriger eingeschätzt als in den übrigen drei Call Center-Typen. Drei der 14 Call Center gehören zum Typ IM.

\section{Abb. 2: Prozentanteile der Tätigkeiten für den Typ IM}

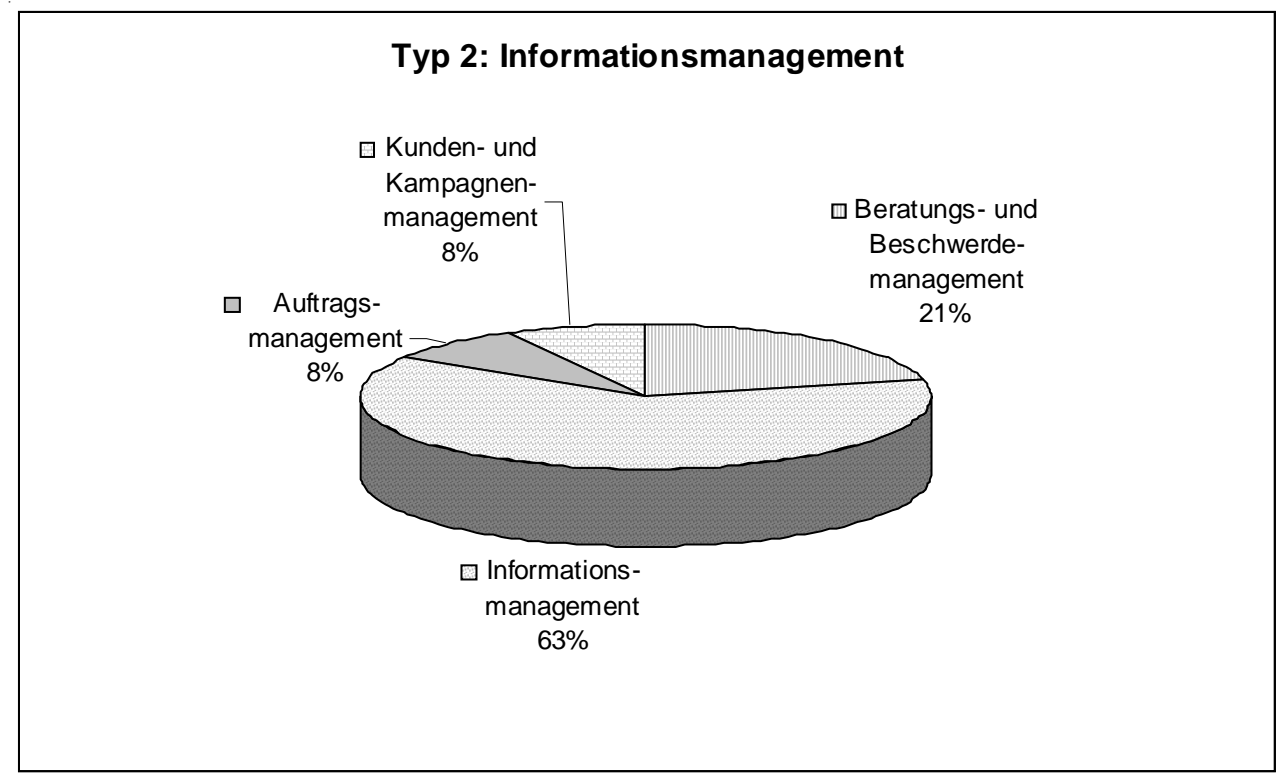

\subsection{Auftragsmanagement}

Die Call Center vom Typ AM bieten Dienstleistungen im Bereich eines Bestell- oder Buchungsservices an. Sie verkaufen Produkte oder Dienstleistungen und nehmen Aufträge entgegen. Die Kunden wenden sich bewusst an diese Call Center und kennen meist die Marken und Aktivitäten des Unternehmens. Nebentätigkeiten sind das IM (22\%) und das BBM (17\%). Ein sehr geringer Anteil bezieht sich auf das aktive KKM. Es werden fast ausschließlich Inbound-Anrufe getätigt (99\%). Die Dauer der Telefongespräche ist eher kurz (durchschnittlich 2,7 Minuten). Die Ganzheitlichkeit der Aufgabe und der Tätigkeitsspielraum werden als mittelmäßig eingestuft, sind jedoch signifikant höher als im Typ IM. Vier der 14 Call Center gehören zum Typ AM. 
Abb. 3: Prozentanteile der Tätigkeiten für den Typ AM

\section{Typ 3: Auftragsmanagement}

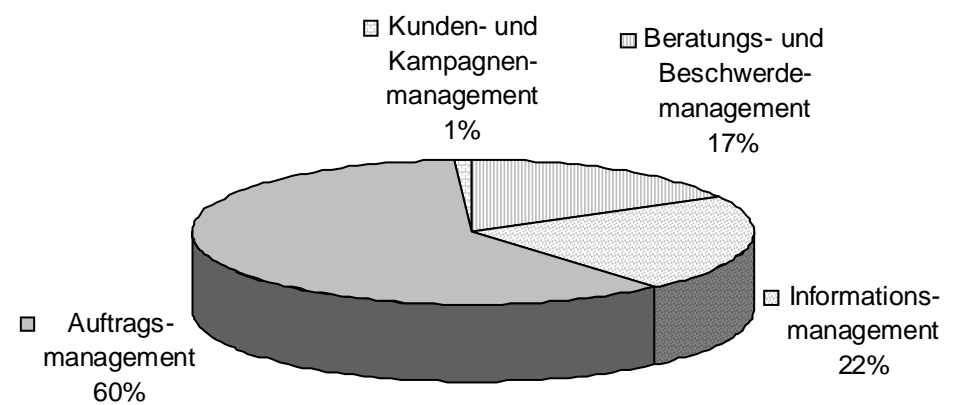

\subsection{Kunden- und Kampagnenmanagement}

Die Hauptaufgaben des Typs KKM liegen im aktiven Management von Kundenbeziehungen (=Leistungen, die auf bestehende Kunden zielen, wie z. B. Kundenrückgewinnung oder aktive Kundenbetreuung) sowie im Kampagnenmanagement (= alle Dienstleistungen, die eine

Abb. 4: Prozentanteile der Tätigkeiten für den Typ KKM

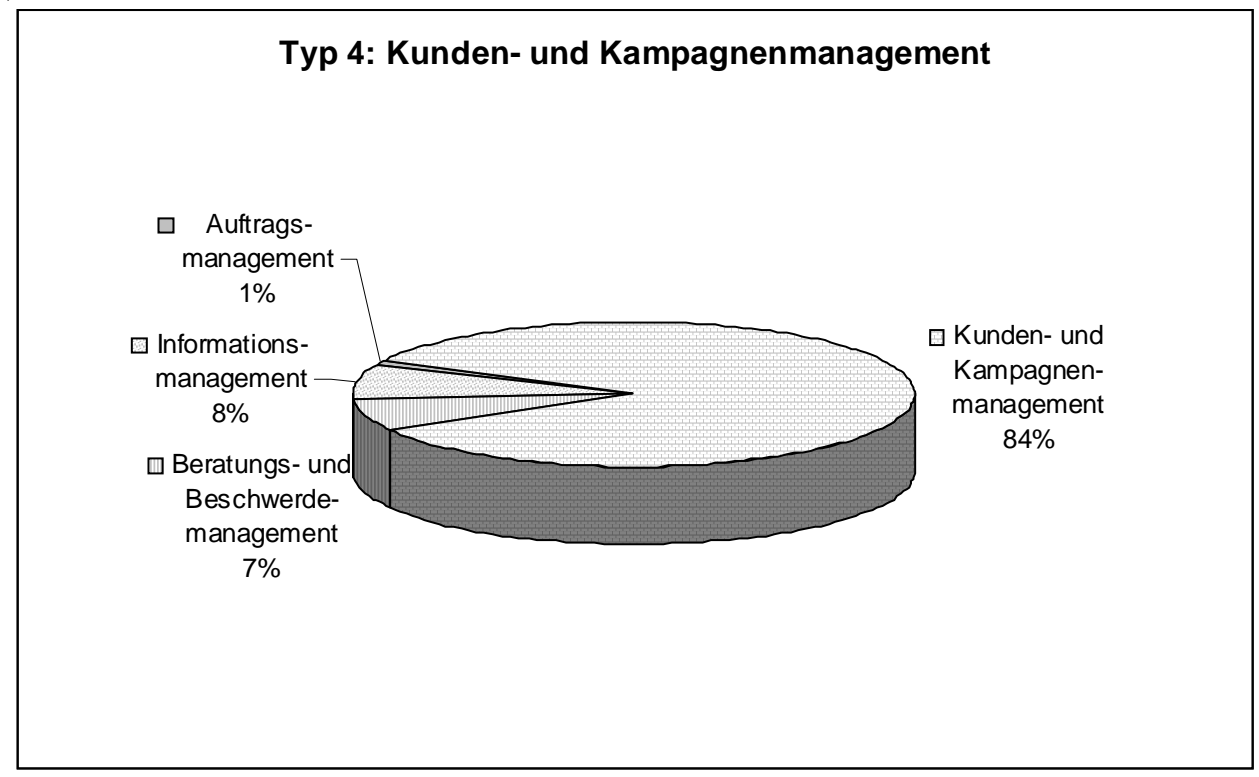


definierte Aufgabe/Kampagne verfolgen, deren Laufzeit zeitlich beschränkt ist). Die Nebentätigkeiten sind das IM (8\%) und das BBM (7\%). Da es sich um Outbound-Call Center handelt (99\%), findet kaum Auftragsmanagement statt. Die Dauer der Telefongespräche liegt durchschnittlich bei 4 Minuten und ist somit signifikant höher als bei den Typen IM und AM, jedoch signifikant niedriger als im Typ BBM. Die Ganzheitlichkeit der Aufgabe wird eher hoch eingeschätzt, der Tätigkeitsspielraum als mittelmäßig eingestuft. Beide Bereiche sind signifikant stärker ausgeprägt als im Typ IM. Vier der 14 Call Center gehören zum Typ KKM.

\section{Personalselektion}

\subsection{Personalstruktur}

Es gibt zahlreiche Gründe, in einem Call Center zu arbeiten. Viele CCA suchen ganz einfach eine kommunikative Herausforderung in einem jungen Umfeld, welche ihnen den Kontakt zu Menschen ermöglicht. Oder es sind Personen, welche begleitend zu ihrer Ausbildung oder ihren Verpflichtungen als Mutter, noch etwas Geld verdienen möchten. In unserer Studie zeigte sich, dass der wichtigste Grund zur Stellenwahl das junge, lebendige Umfeld ist, gefolgt vom Wunsch, das Bekannte zurück zu lassen und etwas Neues kennen zu lernen.

1. Der Wunsch, in einem jungen lebendigen Umfeld zu arbeiten.

2. Der Wunsch, etwas Neues kennen zu lernen.

3. Die Möglichkeit zum telefonischen Kundenkontakt.

4. Die Suche nach einer interessanten Tätigkeit.

5. Der Wunsch nach netten, sympathischen ArbeitskollegInnen.

Im Typ BBM und IM stehen vor allem das junge, lebendige Umfeld und das Interesse, Neues kennen zu lernen im Vordergrund, während im Typ AM vor allem der telefonische Kundenkontakt und die interessante Tätigkeit wichtige Gründe zur Stellenwahl sind. Im KKM sind die flexiblen Arbeitszeiten der wichtigste Motivationsgrund.

Die Personalstruktur in unserer Stichprobe unterscheidet sich nicht grundsätzlich von derjenigen in deutschen Call Centern. In erster Linie arbeiten junge Frauen mit einem Berufsabschluss in Call Centers. Unterschiede gibt es jedoch zwischen den Call Center-Typen (Baumgartner/Udris 2004b und Tabelle 1).

\section{Tab. 1: Statistische Daten zur Gesamtstichprobe und den vier Call Center-} Typen*

\begin{tabular}{|c|c|c|c|c|c|}
\hline & $\begin{array}{c}\text { Gesamt- } \\
\text { stichprobe } \\
\text { (14) }\end{array}$ & BBM (3) & IM (3) & AM (4) & KKM (4) \\
\hline$\varnothing$ Anzahl CCA pro CC1 & $\begin{array}{c}47 \\
(8-130)\end{array}$ & $\begin{array}{c}90 \\
(57-130)\end{array}$ & $\begin{array}{c}63 \\
(20-92)\end{array}$ & $\begin{array}{c}35 \\
(15-53)\end{array}$ & $\begin{array}{c}14 \\
(8-22)\end{array}$ \\
\hline Geschlecht der CCA der Stichprobe & $\begin{array}{l}67 \% \mathrm{w} \\
33 \% \mathrm{~m}\end{array}$ & $\begin{array}{l}47 \% \mathrm{w} \\
53 \% \mathrm{~m}\end{array}$ & $\begin{array}{l}68 \% \mathrm{w} \\
32 \% \mathrm{~m}\end{array}$ & $\begin{array}{l}87 \% \mathrm{w} \\
13 \% \mathrm{~m}\end{array}$ & $\begin{array}{l}73 \% \mathrm{w} \\
27 \% \mathrm{~m}\end{array}$ \\
\hline $\begin{array}{l}\varnothing \text { Alter der CCA der Stichprobe in } \\
\text { Jahren }^{1}\end{array}$ & $\begin{array}{c}31 \\
(19-69)\end{array}$ & $\begin{array}{c}26 \\
(19-52)\end{array}$ & $\begin{array}{c}31 \\
(20-53)\end{array}$ & $\begin{array}{c}30 \\
(19-46)\end{array}$ & $\begin{array}{c}40 \\
(20-69)\end{array}$ \\
\hline $\begin{array}{l}\varnothing \text { Dauer der CCA im CC tätig in } \\
\text { Monaten }^{1}\end{array}$ & $\begin{array}{c}26 \\
(2-240)\end{array}$ & $\begin{array}{c}25 \\
(3-92)\end{array}$ & $\begin{array}{c}20 \\
(2-96)\end{array}$ & $\begin{array}{c}40 \\
(4-240)\end{array}$ & $\begin{array}{c}17 \\
(2-66)\end{array}$ \\
\hline $\begin{array}{l}\varnothing \text { Fluktuationsrate der CCA im Jahr } \\
2001^{1}\end{array}$ & $\begin{array}{c}21 \% \\
(8-50 \%)\end{array}$ & $\begin{array}{c}11 \% \\
(8-18 \%)\end{array}$ & $\begin{array}{c}27 \% \\
(10-40 \%)\end{array}$ & $\begin{array}{c}21 \% \\
(10-28 \%)\end{array}$ & $\begin{array}{c}24 \% \\
(10-50 \%)\end{array}$ \\
\hline
\end{tabular}

* Anzahl Call Center in Klammern); ${ }^{1}$ Mittelwerte (Range: Min./Max. in Klammern) 


\subsection{Kompetenzanforderungen}

In Beiträgen zum Thema Call Center werden oftmals auch die Anforderungen, die an CCA gestellt werden, angesprochen (u. a. Dollinger 1999; Gerber-Rüegg 2001, 2003; Rowold/ Baumgartner, in Vorbereitung). In der Studie CALL sollten die befragten CCA angeben, welche Kompetenzen ihrer Meinung nach für ihre Arbeit besonders wichtig sind. In der Tabelle 2 sind die sieben Faktoren, welche nach der Faktorenanalyse extrahiert wurden, inhaltlich wiedergegeben (Baumgartner u.a. 2002).

Tab. 2: Kompetenzanforderungen aus der Sicht der CCA*

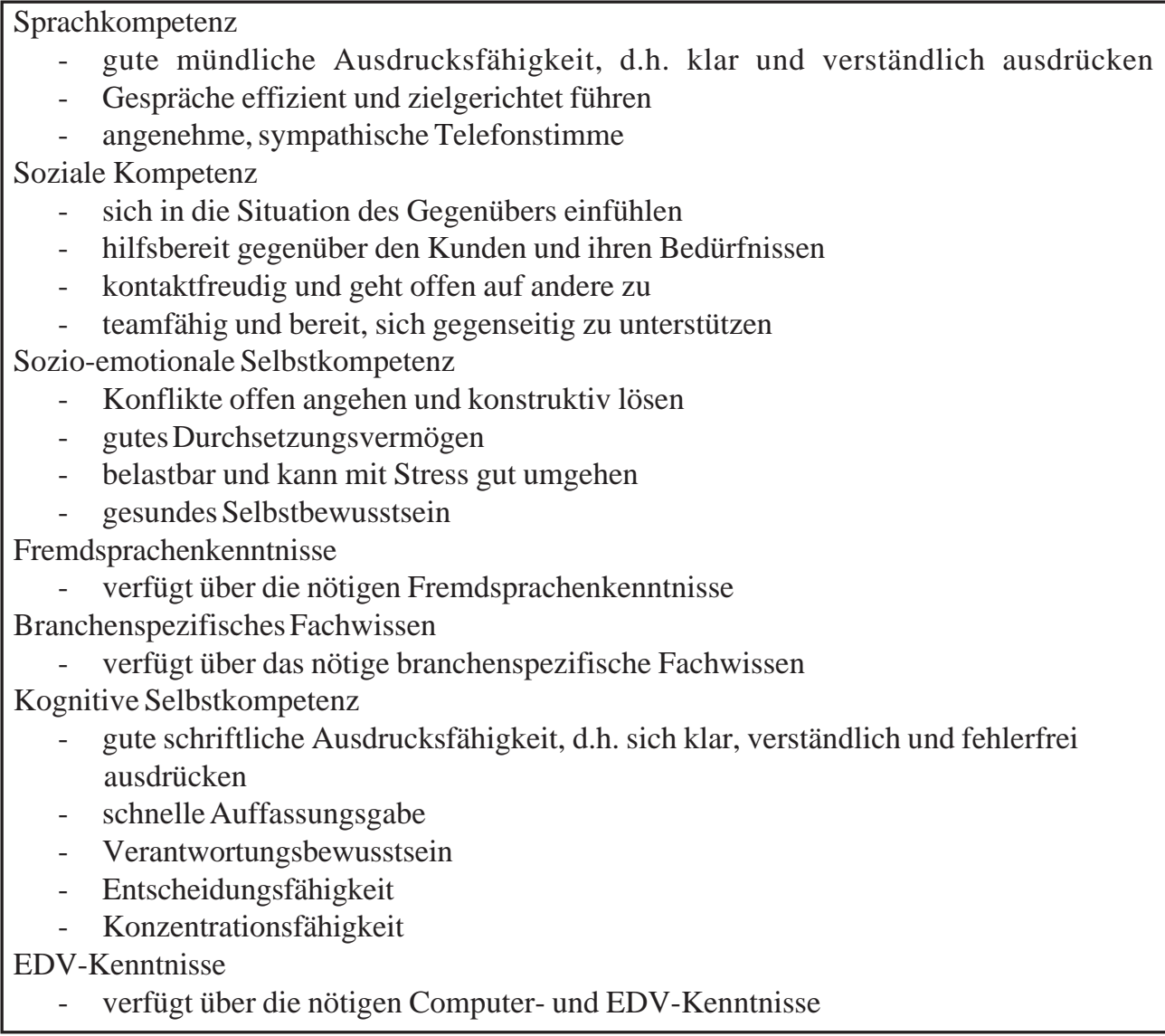

* Ergebnisse der Faktorenanalyse

Die Fähigkeiten, sich mündlich auszudrücken und ein Gespräch effizient und zielgerichtet durchzuführen, sowie die Gabe, über eine angenehme Stimme zu verfügen, sind für CCA besonders wichtig (vgl. Tabelle 3). Doch auch soziale Kompetenzen - wie Einfühlungsvermögen, Hilfsbereitschaft, Teamfähigkeit und Kontaktfähigkeit-sind wichtige Voraussetzungen für den Erfolg als CCA. An dritter Stelle steht die so genannte sozio-emotionale Selbstkompetenz. Dazu gehören Konfliktfähigkeit, Belastbarkeit, Durchsetzungsvermögen 
und ein gesundes Selbstbewusstsein. Auch hier gibt es signifikante Unterschiede zwischen den Call Center-Typen (vgl. Tabelle 3).

Tab. 3: Kompetenzanforderungen aus der Sicht der CCA*

\begin{tabular}{|c|c|c|c|c|c|}
\hline & $\begin{array}{c}\text { Gesamtstich-- } \\
\text { probe } \\
(\mathbf{N}=\mathbf{2 4 2})\end{array}$ & $\begin{array}{c}\text { BBM } \\
(\mathbf{N}=\mathbf{8 0})\end{array}$ & $\begin{array}{c}\text { IM } \\
(N=68)\end{array}$ & $\begin{array}{c}\text { AM } \\
(N=62)\end{array}$ & $\begin{array}{c}\text { KKM } \\
(\mathbf{N}=32)\end{array}$ \\
\hline Sprachkompetenz & $\mathrm{m}=4,2 / \mathrm{s}=0.5$ & $\mathrm{~m}=4,1 / \mathrm{s}=0.6$ & $\mathrm{~m}=4,3 / \mathrm{s}=0.5$ & $\mathrm{~m}=4,2 / \mathrm{s}=0.5$ & $\begin{array}{c}\mathrm{m}=4,5 / \mathrm{s}=0.5 \\
>>\mathrm{BBM} \\
>\mathrm{IM} \\
>>\mathrm{AM}\end{array}$ \\
\hline Soziale Kompetenz & $\mathrm{m}=4,1 / \mathrm{s}=0.6$ & $\mathrm{~m}=4,0 / \mathrm{s}=0.6$ & $\mathrm{~m}=4,0 / \mathrm{s}=0.7$ & $\mathrm{~m}=4,0 / \mathrm{s}=0.6$ & $\begin{array}{c}\mathrm{m}=4,4 / \mathrm{s}=0.6 \\
>>\mathrm{BBM} \\
>>\mathrm{IM} \\
>\mathrm{AM}\end{array}$ \\
\hline $\begin{array}{l}\text { Sozio-emotionale } \\
\text { Selbstkompetenz }\end{array}$ & $\mathrm{m}=4,0 / \mathrm{s}=0.6$ & $\mathrm{~m}=3,8 / \mathrm{s}=0.6$ & $\mathrm{~m}=4,0 / \mathrm{s}=0.6$ & $\mathrm{~m}=4,0 / \mathrm{s}=0.4$ & $\begin{array}{c}\mathrm{m}=4,1 / \mathrm{s}=0.6 \\
>\mathrm{BBM}\end{array}$ \\
\hline Fremdsprachenkenntnisse & $\mathrm{m}=3,8 / \mathrm{s}=1.0$ & $\mathrm{~m}=3,8 / \mathrm{s}=0.9$ & $\mathrm{~m}=3,6 / \mathrm{s}=1 \cdot 0$ & $\begin{array}{c}\mathrm{m}=4,2 / \mathrm{s}=0.7 \\
>>\text { BBM } \\
>>\text { IM }\end{array}$ & $\begin{array}{c}\mathrm{m}=3,0 / \mathrm{s}=1.1 \\
<<\mathrm{BBM} \\
\quad<<\mathrm{IM} \\
<<\mathrm{AM}\end{array}$ \\
\hline $\begin{array}{l}\text { Branchenspezifisches } \\
\text { Fachwissen }\end{array}$ & $\mathrm{m}=3,7 / \mathrm{s}=1.0$ & $\begin{array}{c}\mathrm{m}= \\
=3,9 / \mathrm{s}=0.9 \\
>>\mathrm{IM} \\
>\mathrm{KKM}\end{array}$ & $\mathrm{m}=3,4 / \mathrm{s}=0.9$ & $\begin{array}{c}\mathrm{m}=3,8 / \mathrm{s}=1.1 \\
>\mathrm{IM}\end{array}$ & $\mathrm{m}=3,4 / \mathrm{s}=1.0$ \\
\hline Kognitive Selbstkompetenz & $\mathrm{m}=3,6 / \mathrm{s}=0.6$ & $\mathrm{~m}=3,5 / \mathrm{s}=0.7$ & $\mathrm{~m}=3,6 / \mathrm{s}=0.7$ & $\mathrm{~m}=3,7 / \mathrm{s}=0.5$ & $\mathrm{~m}=3,7 / \mathrm{s}=0.6$ \\
\hline EDV-Kenntnisse & $\mathrm{m}=3,3 / \mathrm{s}=0.9$ & $\begin{array}{c}\mathrm{m}=3,5 / \mathrm{s}=0.9 \\
>>\mathrm{AM}\end{array}$ & $\mathrm{m}=3,4 / \mathrm{s}=0.8$ & $\mathrm{~m}=3,1 / \mathrm{s}=0.8$ & $\begin{array}{c}\mathrm{m}=2,7 / \mathrm{s}=1.0 \\
<<\mathrm{BBM} \\
<<\mathrm{KKM} \\
<\mathrm{AM}\end{array}$ \\
\hline
\end{tabular}

* Mittelwertvergleiche; $\mathrm{m}=$ Mittelwert auf einer Skala von 1 - 5; s = Standardabweichung

$<</>>=\mathrm{p}<0.01$ (hoch signifikant); $</>=\mathrm{p}<0.05$ (signifikant)

Die Call Center-Betreiber sollten angeben, in welche der erwähnten Kompetenzanforderungen auch investiert wird. Am meisten wird in das branchenspezifische Fachwissen und in die EDV-Kenntnisse investiert. Erst an dritter Stelle folgt die Sprachkompetenz. Sozio-emotionale Selbstkompetenz sowie kognitive Selbstkompetenz werden kaum unterstützt (Baumgartner u.a. 2002). Zudem gibt es Unterschiede zwischen den Call Center-Typen, so wird z. B. in den Typen KKM und IM stärker in die Entwicklung der Sprachkompetenz investiert, als dies in den beiden anderen Call Center-Typen der Fall ist.

Ein kurzer Blick in die Zukunft soll aufzeigen, mit welchen Veränderungen Call CenterBetreiber und deren Personal in Zukunft konfrontiert werden. Betrachtet man die gegenwärtige Entwicklung der Branche, so zeichnen sich klare Tendenzen ab, welche die Bedeutung des Menschen in der Interaktion mit der Technik noch verstärken. So ist beispielsweise das Anrufvolumen unter dem Einfluss des E-Commerce weiter angestiegen. Die Kontakte haben sich vom Telefon vermehrt auch auf E-Mails, Voice-Mails und SMS verlagert. Die Anforderungen an das Personal sind angesichts der zunehmenden Komplexität der zu lösenden Probleme angestiegen (Gerber-Rüegg 2003). Als Folge finden Bestrebungen statt, Ausbildungsgänge für CCA, CCT und CCM zu forcieren und zu vermarkten. 
Folgende Trends für die Anforderungen an CCA lassen sich feststellen:

- Die Technik setzt den Rahmen der Leistungserbringung durch den Menschen.

- Die Anwendung komplexer Computerprogramme und anderer Technik wird zur Voraussetzung der Arbeitsleistung.

- Die Problemlösungs- und Kommunikationskompetenz - inklusive der schriftlichen Ausdrucksfähigkeit der Mitarbeitenden - gewinnt an Bedeutung.

- Fremdsprachenkenntnisse und interkulturelle Kompetenzen werden noch wichtiger.

- Call Center werden zu einer Schlüsselfunktion im betrieblichen Wissensmanagement und Marketing.

- Einfachere Auskünfte und Informationen werden den KundInnen vermehrt in Selbstbedienung angeboten, d.h. der Schwierigkeitsgrad der Aufgaben in den Call Centern nimmt ständig zu.

- Der Call Center-Typ des Beratungs- und Beschwerdemanagements erhält künftig die größte Bedeutung im Inbound-Bereich.

\subsection{Selektionsverfahren}

Da man in den neunziger Jahren noch wenig über die Anforderungen der Call Center-Arbeit wusste, gab es kaum realistische Anforderungsprofile und auch keine spezifischen Verfahren zur Mitarbeiterselektion. Da die Nachfrage nach CCA jedoch sehr groß war, hatte dies zur Folge, dass fast jeder und jede CCA werden konnte, sofern er oder sie gerne telefonierte und über PC-Grundkenntnisse verfügte. Obwohl sich vor allem kaufmännische Angestellte und VerkäuferInnen bewarben, wurden auch immer wieder Coiffeure, Handwerker, Abiturienten, Krankenschwestern, Kindergärtnerinnen usw. rekrutiert. Zudem wurde bei der Rekrutierung kaum berücksichtigt, um was für eine Call Center-Tätigkeit es sich handelte, das heißt, es wurde kaum ein Unterschied gemacht, ob jemand eine Hose verkaufen, ein technisches Handyproblem lösen oder einen Anruf weiterleiten sollte. Will man jedoch einen zukunftsweisenden Weg für die Rekrutierung von CCA einschlagen und sich dadurch auch Marktvorteile sichern, heißt es in erster Linie, sich Klarheit über die Zielsetzungen der Auswahl zu verschaffen sowie Kriterien festzulegen, mit deren Hilfe diese Zielsetzungen erreicht werden können. Für die Call Center-Branche heißt dies, kompetente, motivierte und leistungsstarke CCA zu rekrutieren. Voraussetzungen dafür sind:

- Klarheit über den eigenen Call Center-Typ, d. h. über die im Call Center durchgeführten Tätigkeiten, schaffen.

- Kenntnisse über die daraus resultierenden Anforderungen, die an die CCA gestellt werden, generieren.

- Maßnahmen zur Überprüfung der entsprechenden vorhandenen Kompetenzen bzw. des Potentials der BewerberInnen entwickeln und einsetzen.

Die Punkte 1 und 2 wurden in diesem Beitrag bereits angesprochen und werden daher an dieser Stelle nicht weiter kommentiert. Punkt 3 soll an dieser Stelle kurz erläutert werden. Die Selektionsverfahren in Schweizer Call Centern laufen ähnlich und doch auch sehr unterschiedlich ab. Die Verantwortung liegt entweder beim CCM, CCT oder der Human ResourcesAbteilung. Die folgende Aufzählung zeigt Rekrutierungsmaßnahmen, die in Call Centers eingesetzt werden: Sichtung der Bewerbungsunterlagen, Telefoninterviews, Bewerbungsgespräche (manchmal auch mehrere Gespräche), Besichtigung des Call Center (evtl. Möglichkeit zum Beisitzen bei Telefongesprächen), Gespräch mit anderen CCA und CCT über die Call Center-Arbeit, Persönlichkeitstests durch qualifizierte Personen (Human Resources-Abtei- 
lung), Schnuppern im Call Center (ca. halber Tag), Arbeitssimulationen (Telefontest), Fremdsprachentests, Gruppendiskussionen.

Unterschiede zeigen sich vor allem in der Zusammensetzung und dem Umfang dieser Selektionsinstrumente. Es ist folglich schwierig, ein einheitliches Bild über die Rekrutierungspraktiken zu geben. Lediglich die Führung der BewerberInnen durch das Call Center und mindestens ein Bewerbungsgespräch werden in sämtlichen untersuchten Call Centern eingesetzt. Es fällt jedoch auf, dass der Rekrutierungsaufwand in den Typen BBM und IM eher hoch ist, während er im Typ AM am geringsten ausfällt. Einerseits geht es bei der Selektion geeigneter CCA darum, die gewünschten Kompetenzen und Fähigkeiten möglichst reliabel zu erfassen. Andererseits muss auch den Bewerbenden ein annähernd realistisches Bild über die Tätigkeit vermittelt werden, damit die in der Folge angetroffene Realität auch möglichst gut mit den Erwartungen übereinstimmt. Die im Vorfeld bestehenden Erwartungen an die Stelle und die vorgefundene Realität stimmt in der Retrospektive bei den CCA im Typ AM am besten überein, während sie bei den AgentInnen im Typ IM am wenigsten übereinstimmt (Baumgartner u.a. 2002). Unerfüllte Hoffnungen und Erwartungen führen zwangsläufig zu Frustration und Resignation oder einem Stellenwechsel.

\section{$4 \quad$ Personalentwicklung}

Das schnelle Wachstum der Call Center-Branche hatte dazu geführt, dass in den letzten Jahren vor allem junge, branchenfremde CCA eingestellt wurden. Viele CCA waren den Belastungen eines Call Center-Arbeitsplatzes daher nicht gewachsen und verließen das Unternehmen oder wechselten intern die Stelle. Hinzu kommt die rasche Entwicklung in der Technik und des Call Center-Marktes in Richtung multimedialer Contact Centers, welche neue Herausforderungen aber auch Risiken für die Call Center-Betreiber sowie für die Mitarbeitenden bergen. Die Entwicklung von kompetenten und leistungsfähigen CCA ist jedoch mit hohem zeitlichen und finanziellen Aufwand verbunden. Die Betreiber scheuen oftmals diesen Aufwand, da die Rentabilität der Investitionen angesichts der hohen Fluktuationsraten kaum gewährleistet ist. Zudem stellen sie sich berechtigterweise die Frage, inwiefern CCA qualifiziert und weitergebildet werden sollen, wenn in den für Call Center typischen flachen Hierarchiestrukturen kaum Aufstiegsmöglichkeiten bestehen. Aufstiegschancen ergeben sich fast ausschließlich, wenn das Call Center wächst. Grundsätzlich gibt es im Call Center drei Entwicklungsmöglichkeiten (Schmitz 2001; Haas 2000):

- Bei der vertikalen Entwicklung werden Führungspositionen in Call Centern oftmals intern besetzt. CCA werden zu CCT. Die geförderten CCT verfügen meist über allgemeine Unternehmenskenntnisse und gute Fachkompetenzen, es mangelt ihnen jedoch oft an den nötigen Führungskompetenzen.

- Bei der horizontalen Entwicklung geht es vor allem um die Übernahme neuer, zusätzlicher Aufgaben durch die CCA. Beispiele dafür sind: Betreuung neuer Produkte oder Kundensegmente, Projektarbeit, Backoffice-Tätigkeiten, Schulungs- und Betreuungsaufgaben, Stabsaufgaben, EDV-Spezialist. Dies kann im Sinne eines ,job enrichment“, aber auch als Übernahme einer neuen Funktion stattfinden.

- Als dritte Möglichkeit kann die Call Center-Tätigkeit als Karriere-Sprungbrett innerhalb einer Firma gesehen werden. Diese Möglichkeit besteht jedoch nur bei Inhouse-Call Center, welche die CCA als Rekrutierungspotential für Fachabteilungen sehen. 
Das arbeitswissenschaftliche Interesse am Thema Personalmanagement in Call Centers hat in den letzten Jahren zugenommen (Bittner/Schietinger/Weinkopf 2002; Pardini/Schöni 2003; Rowold/Baumgartner, in Vorbereitung; CCALL; FREQUENZ). Dabei ist ersichtlich, dass die drei wichtigsten Maßnahmen zur Kompetenzentwicklung der CCA deren Initialausbildung, die internen oder externen Weiterbildungen und das individuelle Coaching sind.

\subsection{Ausbildung}

Unter dem Patronat des Schweizerischen Call Center Verbandes CallNet können in der Schweiz gegenwärtig innerhalb einiger Monate die folgenden Diplomgänge absolviert werden: Call Center AgentIn, Call Center SupervisorIn (offizielle Bezeichnung für CCTeamleiterInnen), Call Center ManagerIn, Customer Relationship Management (CRM).

Die Diplomgänge werden zwar regelmäßig durchgeführt, die Nachfrage ist jedoch nicht sehr groß. Gerade wenig verdienende CCA können sich die Ausbildung nicht leisten und sind auf eine Finanzierung durch den Arbeitgeber angewiesen. Letztere sind jedoch kaum an diplomierten AgentInnen interessiert. Sie investieren ihr Geld lieber in deren interne branchen- und produktspezifische Ausbildung, welche zielgerichtet auf die eigenen Bedürfnisse angepasst werden kann. Für Firmen stellt somit die außerbetriebliche Ausbildung zum CCA keine Alternative zur firmeninternen Ausbildung dar. Denn so verschieden die Ansprüche der jeweiligen Firmen an ihre CCA sind, so individuell muss deren Ausbildung gestaltet werden. In der Regel werden zu Beginn der Anstellung die neuen Mitarbeitenden in internen Kursen auf ihre Arbeit vorbereitet und mehr oder weniger schnell am Telefon eingesetzt. Das Prinzip der Paten, erfahrene CCA, welche die neuen CCA während der Ausbildungszeit betreuen, scheint sich zudem allgemein durchgesetzt zu haben. Ansonsten gestaltet sich die Ausbildung zum CCA in den Schweizer Call Centern recht unterschiedlich.

Im Schnitt dauert die Initialausbildung zirka vier Wochen. Die CCA werden meist intern zu den Schwerpunktthemen Fachwissen, Computersysteme und Gesprächsführung ausgebildet. Handelt es sich um Call Center, welche projektbezogen arbeiten (primär im Typ KKM), werden die CCA den Projektinhalten entsprechend ausgebildet und bereits nach wenigen Tagen am Telefon eingesetzt. Vertiefte Fachkenntnisse lassen sich auf diese Weise jedoch kaum vermitteln. In den Typen BBM und IM kann die Ausbildung bis zu drei Monaten dauern (Baumgartner/Udris 2003, 2004b).

Die folgende Aufzählung zeigt Maßnahmen für die Initialausbildungen auf, welche in Schweizer Call Centern eingesetzt werden: Interne Schulungen (seltener externe), Paten, Einarbeitung „on-the-job“, Coaching durch Vorgesetzte, Individueller Ausbildungsplan, Unterlagen zum Selbststudium, Hospitation in anderen Abteilungen, (um Firma und Prozesse besser kennen zu lernen), Rollenspiele (z. B. Simulation von Gesprächsverläufen).

\subsection{Weiterbildung und Entwicklung}

Weiterbildungs- und Entwicklungsmöglichkeiten sind weitere wichtige Aspekte des Personalmanagements. Als Weiterbildungen gelten die von der Firma intern oder extern organisierten Maßnahmen zur Entwicklung der Kenntnisse und Fähigkeiten der CCA entsprechend der an sie gestellten Anforderungen. Als Entwicklung gelten die von der Firma eingeleiteten Maßnahmen, um die CCA auf zukünftige Karriereschritte, d. h. Aufstiegs- oder Umstiegsmöglichkeiten, vorzubereiten. Für die Mitarbeitenden und deren Motivation kann es entscheidend sein, ob sie sich in einer Funktion weiterbilden und entwickeln können, oder ob sie sich als 
CCA lediglich auf einem „Abstellgleis“ befinden, wohin sie manövriert und dann „,vergessen“ wurden. Die Bereitschaft der Unternehmen, ins Humankapital zu investieren und ihre CCA zu fördern, ist heute noch gering, dies entgegen allen Beteuerungen. Daran kann sich nur etwas ändern, wenn sich die konsequente Ausrichtung der Unternehmen auf ihre KundInnen durchsetzt. Dabei kommt den CCA eine Schlüsselrolle zu, welche sich in der Arbeitsplatzbewertung und damit auch in entsprechenden Entwicklungsmaßnahmen niederschlagen müssen.

Die CCA aus dem Projekt CALL schätzen die Weiterbildungs- wie auch die Entwicklungsmöglichkeiten sowie die Unterstützung der Firma für ihre Entwicklung und Weiterbildung als eher gering bis mittelmäßig ein. Gleichzeitig ist jedoch das Interesse der CCA an Entwicklung und Weiterbildung hoch. Vor allem die CCA der Typen BBM und IM sind sehr karrierebewusst. Auffallend ist die Tatsache, dass die CCA im Typ IM meist zum Ziel haben, das Call Center zu verlassen, während in den anderen drei Call Center-Typen eher die Hoffnung besteht, intern Karriere zu machen. Auf weitere Details und Unterschiede zwischen den Call Center-Typen wird im Artikel von Baumgartner und Udris (2004b) eingegangen.

Je größer eine Firma ist und je mehr Abteilungen vorhanden sind, umso besser gestalten sich die Entwicklungs- und Weiterbildungsmöglichkeiten für die CCA, wenn auch nicht intern im Call Center, so doch zumindest in anderen Abteilungen der Organisation. Dass es sich dabei trotzdem um einen Wissensverlust für das Call Center handelt, wird von den Betreibern im Allgemeinen akzeptiert. Von vielen Call Center-Betreibern wird das Call Center lediglich als Übergangslösung angesehen.

In Deutschland wurde von Schietinger und Schroth (2001) darauf hingewiesen, dass sich im Bereich Weiterbildungen ein uneinheitliches Bild zeigt. Auch in der Studie CALL zeigt sich, dass Weiterbildungen in Call Centers ganz unterschiedlich gehandhabt werden. Je nach Call Center-Typ finden Weiterbildungen regelmäßiger oder nur selten statt. So hatten z. B. im Typ BBM 89\% der CCA zwischen 2000 und 2001 eine Weiterbildung zum Thema Umgang mit Kunden, während im Typ AM lediglich 53\% der CCA zu diesem Thema weitergebildet wurden. Angesichts dieser unterschiedlichen Weiterbildungspraktiken, dem nach wie vor fehlenden, einheitlichen Berufsbild, der unterschiedlichen Erwerbsbiografien der CCA und der zu erwartenden technologischen Weiterentwicklungen in der Call Center-Branche spricht einiges für die Notwendigkeit, den individuellen Qualifizierungsbedarf der CCA zu ermitteln und entsprechende Angebote zu entwickeln und einzusetzen.

\subsection{Coaching}

Das individuelle Coaching der CCA ist eine weitere, effiziente Entwicklungs- und vor allem auch Kontrollmaßnahme in Call Centern. Oft wird das Coaching einseitig dazu genutzt, die Effizienz und Leistung der Mitarbeitenden in der Kundenberatung zu steigern, nur selten wird dabei auf die individuellen Wünsche und Bedürfnisse der CCA Rücksicht genommen. Das Coaching umfasst den interaktiven, individuellen Betreuungsprozess, der den CCA hilft, ihre Arbeit effizienter und qualitativ besser zu erledigen. Die Häufigkeiten der Coachings in den Call Centern variieren stark. Zwischen einem Coaching pro Woche und einem Coaching pro Jahr ist praktisch alles möglich. Eher häufig und regelmäßig finden Coachings in den Typen BBM und IM statt. Im Typ BBM gehört das Aufzeichnen von Telefonaten für spätere Coachings zur täglichen Routine. Die Akzeptanz der AgentInnen gegenüber diesen Gesprächsaufzeichnungen ist daher signifikant stärker ausgeprägt als bei den CCA der Typen AM und KKM, wo Coachings eher unregelmäßig und selten durchgeführt werden und sich die CCA mit diesem Kontrollinstrument noch schwer tun. 
Bei den Coachings wird vor allem auf die Gesprächsführung, d. h. auf die Einhaltung von vorgegebenen Gesprächsabläufen, sowie die Freundlichkeit und die Kundenorientierung der CCA geachtet. Fachliche Inhalte werden kaum berücksichtigt. Auch sozio-emotionale Inhalte werden nur dann besprochen, wenn ein konkretes Problem während des Gesprächs aufgetaucht ist. Das Fachwissen und der Umgang mit Kunden sind jedoch diejenigen Themen, die sich die Agenten für das Coaching am stärksten wünschen. Fachliche Unsicherheiten und Wissenslücken können belastend sein, zumal die Anforderungen der KundInnen immer größer und deren Vorwissen immer besser werden. Auch der Umgang mit den unterschiedlichen Emotionen der KundInnnen ist eine stetige Herausforderung (Dormann/Zapf/Isic 2003). Eine Ausnahme stellt der Typ KKM dar. Dort wünschen sich die CCA weniger Coaching in fachlicher Hinsicht, als vielmehr in der Gesprächsführung sowie im Umgang mit Kunden und im Selbst-Management. Die CCA werden dort zu Beginn vorwiegend projekt- und produktbezogen ausgebildet und eingesetzt. Sie verfügen dementsprechend über das nötige Fachwissen. Es besteht also bis zu einem gewissen Grad eine Diskrepanz zwischen den Coachinginhalten und den Coachingwünschen der CCA. Coaching ist zum gegenwärtigen Zeitpunkt sicherlich ein sinnvolles Mittel, um die Qualität der Kundengespräche sicherstellen zu können. Trotzdem könnte das Coaching auch noch sozio-emotionale Aspekte (Umgang mit Kunden) oder fachliche Aspekte beinhalten. Die Call Center-Betreiber versuchen, diese Themen jedoch primär durch andere Gefäße, wie Teamsitzungen und Weiterbildungen, zu vermitteln. Diese haben vor allem den Vorteil, dass sie effizienter und ökonomischer sind. Gruppencoaching bzw. das gegenseitige Coaching durch die CCA werden kaum praktiziert. Die meisten Call Center-Betreiber sind der Meinung, dass das Coaching in den Aufgabenbereich der CCT gehört. Damit wird in Kauf genommen, dass die CCT sehr viel Zeit für die Coachings aufwenden müssen und oft, wenn sie quantitativ überlastet sind, das Coaching sogar wegfallen lassen.

\section{Schlussfolgerungen}

Personalentwicklung hat nicht nur mit Aus- und Weiterbildung zu tun. Auch Maßnahmen im Bereich der Arbeitsorganisation und-gestaltung, wie z. B. teilautonome Arbeitsgruppen, ,job rotation“ oder ,job enrichment“, können zur Förderung individueller Kompetenzen verändert und angepasst werden. Solche Maßnahmen werden jedoch, in den untersuchten Call Centers, kaum eingesetzt. Nur selten finden beispielsweise größere Projektarbeiten statt, bei denen CCA aktiv mitgestalten und ihr Wissen einbringen können. Auch eine größere Autonomie der CCA, wie dies in teilautonomen Arbeitsgruppen, dezentralen Arbeitsgruppen oder bei der Telearbeit der Fall ist, wird kaum als Möglichkeit in Betracht gezogen. Statt sich auf Aspekte der Tätigkeit und der Arbeitsorganisation zu konzentrieren, richten die Call Center-Betreiber ihren Fokus auf die Optimierung der Prozessabläufe und nehmen damit eine noch stärkere Rigidität des Handlungsspielraumes der CCA in Kauf.

Um der schleichenden Monotonie etwas entgegenzuhalten, entwickeln die Betreiber unterschiedliche Strategien. Einige stellen nur noch Teilzeit-Mitarbeitende ein. Andere gehen grundsätzlich davon aus, dass es sich bei der Call Center-Tätigkeit um eine Übergangslösung - im Sinne eines Karrieresprungbretts - handelt und nehmen die hohe Fluktuationsrate als gegeben hin. Wiederum andere versuchen, die CCA bei kleineren Projekten oder Back OfficeAufgaben einzusetzen, um den Abwechslungsreichtum etwas zu vergrößern. Ergebnisse aus 
der CALL-Studie zeigen, dass in Call Centers, welche Arbeitsgestaltungsmaßnahmen zur Kompetenzentwicklung ihrer CCA gezielt einsetzen, die Belastungen und die Fluktuationstendenz signifikant niedriger sind, während Arbeitszufriedenheit, Commitment und Wohlbefinden signifikant größer sind als in anderen Call Centers (Baumgartner u.a. 2002).

Kompetenzentwicklung im Call Center ist folglich als systematischer Lernprozess zu verstehen, der darauf zielt, die Fähigkeiten der CCA auf mehreren Gebieten aus- und weiterzubilden und diese zu ihrem eigenen Erfolg und dem des Call Centers einzusetzen. So können CCA durch die Übernahme von verschiedenen Entwicklungsrollen, jedoch innerhalb ihrer Funktion, sich nach und nach ihre Kompetenzen in fachlicher (Ablauf-, Produkte- und Systemwissen), methodischer (Sprachen, Gesprächsführung, Projektarbeit, Informationsverarbeitung, neue Medien etc.) und sozialer (Teamfähigkeit, Kommunikationsfähigkeit, Einfühlungsvermögen etc.) Hinsicht erweitern (Schietinger/Schroth 2001).

\section{Literatur}

Baumgartner, Marc, Katrin Good, Ivars Udris (2002): CALL - Call Centers in der Schweiz: Psychologische Untersuchungen in 14 Organisationen. Eidgenössische Technische Hochschule, Institut für Arbeitspsychologie. Zürich. www.call.ifap.ethz.ch

Baumgartner, Marc, Ivars Udris (2003): Personalentwicklung in Schweizer Call Centers: Neue Anforderungen an Kompetenzen und Arbeitsorganisation; in: Wirtschaftspsychologie, 5, 1, 168 170

Baumgartner, Marc, Ivars Udris (2004a): Call Center Arbeit. Die Menschen hinter den Telefonstimmen; in: Safety-Plus-Schweizer Fachzeitschrift für Arbeitssicherheit und Gesundheitsschutz, 1, 54-56

Baumgartner, Marc, Ivars Udris (2004b): Eine typologische Betrachtung zu Arbeitstätigkeit, Personalentwicklung und Befinden des Personals in Call Centers; in: Zeitschrift für Arbeitswissenschaft, 58, $1,19-28$

Bittner, Susanne, Marc Schietinger, Claudia Weinkopf (2002): Zwischen Kosteneffizienz und Servicequalität - Personalmanagement in Call Centern und im Handel. München

Brasse, Claudia u.a. (2002): AKL-Typologie. Ein empirischer Ansatz zur Typologisierung von Call Centern. Dortmund

CCALL: Erfolgreich und gesund arbeiten im Call Center. Forschungsprojekt: [on-line] www.ccall.de

Dollinger, Anna (1999): Psychologie einsetzen, erfolgreich Mitarbeiter gewinnen-Qualitätsorientierte Mitarbeiterauswahl im Call Center; in: Kirsten Schrick, Anna Dollinger (Hg.): Das innovative Call Center. München, 92-115

Dormann, Christian, Dieter Zapf, Amela Isic (2002): Emotionale Arbeitsanforderungen und ihre Konsequenzen bei Call Center-Arbeitsplätzen; in: Zeitschrift für Arbeits- und Organisationspsychologie, 46, 4, 201-215

FREQUENZ: Personalmanagement - Call Center und Handel. Forschungsprojekt: [on-line]

http://iat-info.iatge.de

Geier, Antja, Gerald Groh, Georg Grass (2001): Referenzprozesse und Rollenkonzepte im Customer Care Center (INCCA AP3). Stuttgart

Gerber-Rüegg, Julia (2001): Call Center - Arbeitsplatz der Zukunft? Zürich

Gerber-Rüegg, Julia (2003): Arbeitszufriedenheit, Motivation und Wohlbefinden. Erkenntnisse aus der Studie „CALL - Call Centers in der Schweiz“ des Instituts für Arbeitspsychologie der ETH Zürich. Zürich

Grebner, Simone u.a. (2003): Working conditions, well-being, and job-related attitutes among call centre agents; in: European Journal of Work and Organizational Psychology, 12, 4, 341-365 
Haas, Sabine (2000): Anforderungswechsel und Karriereplanung im Call Center; in: Rainer Wieland, Karin Scherrer (Hg.): Arbeitswelten von morgen. Wiesbaden, 148-157

Metz, Anna-Marie, Heinz-Jürgen Rothe, Mirko Degener (2001): Belastungsprofile von Beschäftigten in Call Centers; in: Zeitschrift für Arbeits- und Organisationspsychologie, 45, 124-135

Pardini, Giorgio, Walter Schöni (2003): Arbeit und Berufsperspektiven in Call Centers; in: Widerspruch, 45, 135-147

Richter, Peter, Uwe Debitz, Frank Schulze (2002): Diagnostik von Arbeitsanforderungen und kumulativen Beanspruchungsfolgen am Beispiel eines Call Centers; in: Zeitschrift für Arbeitswissenschaft, 1-2, 67-76

Rowold, Jens, Marc Baumgartner (in Vorbereitung): Technozentrierte vs. kundenorientiert-psychologische Leistungsdindikatoren von Inbound Call Center Agents. Eingereicht bei der Zeitschrift für Personalpsychologie

Scherrer, Karin (2001): Dauerarbeitsplatz Call Center: Gesundheitsförderliche Arbeitsgestaltung senkt Fluktuation und Krankenstand; in: Bernhard Badura, Martin Litsch, Christian Vetter (Hg.): Fehlzeiten-Report 2000. Zukünftige Arbeitswelten: Gesundheitsschutz und Gesundheitsmanagement. Berlin, 61-79

Schietinger, Marc, Jochen Schroth (2001): Qualifikation und Qualifizierung von Call Center-Agents: Erste Ergebnisse aus der Basiserhebung. Forschungsprojekt FREQUENZ [on-line]. http:// www.bundes-bonn.de

Schmitz, Eva (2001): Fortbildung in Call Centern; in: Erika Regnet, Laila M. Hofmann (Hg.): Trends, Inhalte und Methoden der Personalentwicklung in Unternehmen. Göttingen

Timm, Elke (2003): Arbeit im Call Center - Tätigkeitsstrukturen, Belastungen und Ressourcen. Unveröff. Dissertation. Universität Wuppertal

Udris, Ivars, Marc Baumgartner (2003): Telefonarbeit in Schweizer Call Centers: Eine typologische Betrachtung zur Arbeitstätigkeit und zum Befinden des Personals; in: Wirtschaftspsychologie, 5, 1, 162-165

www.persoenlich.com (Online-Portal der Schweizer Kommunikationswirtschaft): Call Center: Die fetten Jahre sind vorbei (19.3.2003)

Anschrift der Verfasser:

Lic. phil. Marc Baumgartner

Prof. Dr. Ivars Udris

ETH Zürich

Institut für Arbeitspsychologie

Nelkenstrasse 11

CH-8092 Zürich

mbaumgartner@ethz.ch

iudris@ethz.ch

\section{Schlagwörter: Arbeitsorganisation, Dienstleistungen, Personal / Personalführung, Qualifikation /Aus- und Weiterbildung}

